\title{
Subtypes of depression in cancer patients: an empirically driven approach
}

\author{
Lei Zhu ${ }^{1}$ - Adelita V. Ranchor ${ }^{1}$ - Marije van der Lee ${ }^{2}$ Bert Garssen ${ }^{2}$. \\ Robbert Sanderman ${ }^{1,3}$ • Maya J. Schroevers ${ }^{1}$
}

Received: 7 April 2015 / Accepted: 18 August 2015 / Published online: 5 September 2015

(C) The Author(s) 2015. This article is published with open access at Springerlink.com

\begin{abstract}
Purpose This study aimed to (1) identify subgroups of cancer patients with distinct subtypes of depression before the start of psychological care, (2) examine whether socio-demographic and medical characteristics distinguished these subtypes, and (3) examine whether people with distinct subtypes reported differential courses of depression during psychological care. Method This naturalistic, longitudinal study included cancer patients who sought psychological care at specialized psychooncology institutions in the Netherlands. Data were collected before psychological care (T1) and three (T2) and nine (T3) months thereafter. Latent class analysis was performed to identify depression subtypes in 243 patients at T1.

Results Before starting psychological care, three depressive subtypes were identified, differing in severity and type of symptoms. Class $1(47 \%)$ with mild depression reported mostly concentration and sleep problems and fatigue. Class 2 (41\%), with slightly higher levels of depression, reported similar concentration and sleep problems and fatigue as class 1 , and additionally depressed mood. Class 3 (12\%), with severe depression, reported mainly a depressed mood and, to a lesser extent but still elevated, fatigue and concentration problems. None of socio-demographic and medical
\end{abstract}

Maya J. Schroevers

M.J.Schroevers@umcg.nl

1 Department of Health Psychology, University Medical Centre Groningen, University of Groningen, POB 196, A. Deusinglaan 1, 9700AD Groningen, The Netherlands

2 Helen Dowling Institute, Centre for Psycho-Oncology, Bilthoven, The Netherlands

3 Department of Psychology, Health and Technology, University of Twente, Enschede, The Netherlands characteristics significantly distinguished these subtypes. These subtypes significantly predicted the course of depression over time, with class 1 reporting moderate improvements, class 2 large improvements, and class 3 the largest improvements.

Conclusions Results indicate the presence of three subtypes of depression in cancer patients before starting psychological care. Our findings suggest that psychological interventions could be tailored to respond to the specific subtype of depression experienced by each individual.

Keywords Subtypes of depression - Cancer patients . Psychological care $\cdot$ Latent class analysis

\section{Introduction}

Major depression is characterized by a range of symptoms, with core symptoms of a depressed mood and/or loss of interest/pleasure in normal activities, and also guilty feelings, suicidal thoughts, concentration problems, and sleeping and appetite problems. For a diagnosis of major depressive disorder (MDD), at least five of nine symptoms need to be present, including at least one of the two core symptoms [1]. Depression is one of the most prevalent psychological problems in cancer patients, with prevalence rates of 8 to $24.6 \%[2,3]$. Depression in cancer patients may be associated with lower quality of life [4], less adherence with cancer treatment [5], and prolonged hospitalization [6]. Thus, detection and treatment of depression in cancer patients need to be optimized.

The current broad and heterogeneous definition of depression has proven unsatisfactory, as patients may have various combinations of symptoms. The identification of specific subtypes of depression may increase our understanding of the causes and optimize treatment [7-11]. Efforts have been made 
to identify distinct subtypes of depression, either using a topdown, clinically driven approach or a bottom-up, empirically driven approach. The top-down approach depends on a hypothesized framework by pre-defining subtypes of depression and classifying people based on descriptive clinical data [12]. For instance, depression can be differentiated based on symptom presentation, time of onset characteristics, etiology, and severity [13]. This approach has been criticized because of the limited clinical applicability and problems in identifying and using these subtypes $[11,13,14]$. The bottom-up, empirical approach may help to overcome this problem and identify meaningful heterogeneous depressive subtypes that are based on depressive symptoms that cancer patients actually experience. The empirical approach is designed to discover structure in the absence of a pre-existing hypothesis about the subtypes of depression and relies on advanced statistical techniques to identify subgroups with distinct profiles of symptoms [12]. Using an empirically driven approach, clinicians may identify which subtype of depression each patient has and tailor psychological interventions toward the specific symptoms experienced by each individual.

Specifically, latent class analysis (LCA), as an advanced statistical technique, can be used to classify individuals with similar profiles into heterogeneous classes based on the observed scores of each individual. LCA has been widely used in previous research and demonstrated its superiority in subtyping depression in both people with MDD and general populations [15-23]. A recent systematic review on empirically derived subtypes of depression in adults with MDD concluded that there was no conclusive evidence to demonstrate the number and type of subtypes of depression, given the great diversity in the identified subtypes [11]. Another important observation based on LCA was that both severity and nature of symptoms play an important role in differentiating depressive subtypes [11].

As previous studies mainly focused on general populations and people with MDD [15-23], little is known regarding the subtypes of depression in people with severe comorbid somatic conditions such as cancer. Given the overlap between depressive symptoms and cancer-related symptoms [24], it is important to examine whether the subtypes of depression found in other populations can be identified in cancer patients. Specifically, depressive symptoms including sleep problems, fatigue, loss of appetite, and weight loss can be caused by pathophysiologic processes of cancer and/or concurrent medical treatment (e.g., chemotherapy) [24, 25]. This symptom overlap emphasizes the importance and difficulty of accurately assessing depression in cancer patients [24]. It is important to distinguish between cognitive/affective (e.g., depressed mood, lack of pleasure/interest) and somatic symptoms of depression (e.g., appetite and sleep problems, fatigue) [26]. Using LCA, one study in depressed myocardial infarction (MI) patients found three depression subtypes: a less-severe group $(60 \%)$ reporting mainly a depressed mood with additional concentration problems and fatigue, a moderate-severe group $(23 \%)$ reporting mainly a lack of pleasure with additional concentration problems and fatigue, and a severe group (17\%) reporting almost all cognitive/affective and somatic symptoms of depression [27]. So far, no studies have used an empirically driven approach to identify subtypes of depression in the context of cancer. Thus, it remains unknown regarding the role of cognitive/affective and somatic symptoms in subtyping depression in cancer patients.

In order to fill in these gaps, this study used a bottom-up, empirically driven approach (i.e., LCA) to identify subgroups of cancer patients with distinct subtypes of depression. We focused on cancer patients who were receiving psychological care at a specialized psycho-oncology institution, because these patients would be at risk for clinically elevated depression. We only included patients with clinically elevated levels of depression before the start of psychosocial care. As patients' problems are assessed and diagnosed before psychological care, it seems clinically relevant to know whether patients with heterogeneous subtypes of depression can be identified at that moment. The first aim was to identify patients with distinct subtypes of depression before psychological care. Provided that subgroups of cancer patients with various subtypes of depression can be identified, it is relevant to distinguish these patients. A recent study on a general population has found that several socio-demographic and medical characteristics (e.g., age, gender, education, and physical health) could distinguish people with distinct subtypes of depression [16]. Thus, the second aim was to examine whether these subtypes can be distinguished by the patients' sociodemographic and medical characteristics. As our participants were receiving specialized psycho-oncology care over a 9month period, it can be assumed that, within this context, participants might report changes in depressive symptoms. The changes could be due to the received psychological care or merely reflect a natural adaptation. The third aim was to examine whether patients with distinct subtypes of depression reported differential changes in depression during psychological care.

\section{Method}

\section{Sample and procedure}

Cancer patients who sought psycho-oncological care at one of the seven specialized psycho-oncology institutions in the Netherlands between September 2008 and March 2010 were informed about this study. All seven psycho-oncology institutions in the Netherlands were involved in this study. These institutions aim to help cancer patients, their family, partners, and friends to cope with cancer and offer professional care in 
the form of individual therapies, group therapies, and internet therapies. Eligible participants were (1) diagnosed with cancer and seeking psycho-oncological help, (2) over 18 years, and (3) able to complete questionnaires in Dutch.

We approached 611 patients, of whom 524 agreed to participate and gave written informed consent. The $87 \mathrm{de}-$ cliners did not differ significantly in age or gender from those who agreed. Of these 524 patients, 401 underwent the baseline assessment before the start of psychological care (T1) and 123 dropped out. Of these 401 patients, 384 were included and 17 were excluded. Eight decided not to receive psychological care, and nine did not complete the baseline assessment. The 140 non-participants did not differ significantly from the 384 in age or gender. After 3 months, 278 (72\% of 384) completed the second assessment (T2). After 9 months, 241 (63\% of 384) completed the third assessment (T3). From T1 to T3, 143 dropped out because of illness or other reasons. Compared with the 241 participants who completed the assessment at T3, the 143 dropouts were more likely to be male, have lower education, have received an unfavorable prognosis, and were less likely to undergo surgery $(p<0.05)$. There were no significant differences in baseline depressive symptoms between the 241 sample and the 143 dropouts.

Of the baseline sample of 384 participants, 243 (63\% of 384) reported clinically elevated levels of depression (baseline depressive symptoms $\geq$ cutoff; see "Measures") and were included in the analyses. Of these 243,157 (65\% of 243) completed the second and third follow-up assessments and were used to examine the longitudinal course of depression between patients with distinct subtypes.

\section{Measures}

\section{Socio-demographic and medical characteristics}

A self-report questionnaire was used to collect sociodemographic and medical characteristics (e.g., educational level, cancer type). Educational level was classified into three categories: low (i.e., primary/lower vocational), middle (i.e., secondary/middle vocational), and high (i.e., university/higher vocational). Physical symptoms were assessed by a 10-item checklist (e.g., nausea, headache) based on the physical symptoms subscale of the Rotterdam Symptom Checklist, which has demonstrated good validity and reliability [28]. The 10 -item checklist did not overlap with somatic symptoms that were included in the measure for depression. Each question can be answered on a scale from 1 (none) to 4 (very). Total scores ranged from 10 to 40, with higher scores indicating more physical symptoms. We found a Cronbach's $\alpha$ of 0.74 in the baseline sample of 384 participants.

\section{Psychological care characteristics}

Self-report questionnaires were used to collect psychological care characteristics (i.e., type and duration of psychological care) at T2 and T3. Participants were offered different types of psychological care (i.e., individual, group, or other therapy such as haptonomy). In the questionnaire, patients indicated which therapies they had received. Treatments were categorized as individual, group, individual and group (all with/without other therapy), and other. Patients also indicated whether their psychological care was complete or ongoing at $\mathrm{T} 2$ and $\mathrm{T} 3$.

\section{Depressive symptoms}

The 16-item version of the Center for Epidemiological Studies Depression Scale (CES-D) [29], which excludes the four positively formulated items, has been found to be a more valid measure for depression in cancer patients [30]. We therefore used this version to measure depressive symptoms. Each question can be answered on a scale from $0(<1$ day) to 3 (5-7 days). Total scores ranged from 0 to 48 , with higher scores indicating higher depression levels. A cutoff point of 10 has previously been validated to indicate clinically elevated levels of depressive symptoms [31]. The CES-D has shown good reliability and validity in previous research [29]. Cronbach's $\alpha$ was 0.88 in the 384 participants.

After comparing the 16 CES-D items with the Diagnostic and Statistical Manual of Mental Disorders, Fifth Edition (DSM-5) criteria for MDD [1], we selected 10 CES-D items that matched the DSM-5 criteria: three items referring to "blues, depressed mood, and sadness" were used for assessing depressed mood; two items referring to "everything being an effort and not able to get going" were used for assessing lack of energy/fatigue; one item were used for assessing poor appetite; one item were used for assessing sleep problems; one item referring to "experience life as a failure" were used for assessing feelings of worthlessness; one item referring to "trouble keeping the mind on what I was doing" were used for assessing concentration problems; and one item referring to "talking less" were used as an indication of psychomotor slowing [32]. Two DSM-5 criteria concerning loss of pleasure/interest and suicidal thoughts were not covered. The 10-item CES-D had a Cronbach's $\alpha$ of 0.84 in the baseline sample of 384 participants.

A cutoff point of eight was used for the 10-item CES-D to select cancer patients with clinically elevated depression at baseline. This cutoff matched the percentage of clinical cases based on the cutoff of 10 for the 16-item CES-D: 243 participants (63\% of the total 384 ) reported 10-item CES-D score $\geq 8,257$ participants ( $67 \%$ of the total 384 ) reported 16 -item CES-D scores $\geq 10$, and $98 \%$ of the 243 were also included in the 257 . These 243 participants were included in the analysis. 


\section{Statistical analysis}

LCA was used to identify subgroups of patients with distinct subtypes of depression based on the 10-item CES-D in the 243 participants. All participants fully completed the 10 CES-D items at $\mathrm{T} 1$.

We tested LCA models that ranged from two to five classes. Distinct latent classes represented heterogeneous subgroups of cancer patients with distinct subtypes of depression. Several criteria were used to determine the model that best represented the data. First, we inspected several statistical criteria including the Bayesian information criterion (BIC), the Akaike information criterion (AIC), entropy, and the bootstrapped likelihood ratio test (BLRT). The BIC and AIC are measures of relative fit of different models. The lower the BIC and AIC, the better the model fits. To examine the quality of latent class classification, entropy values were inspected. A higher entropy $(\geq 0.6)$ indicates a better quality of class separation [33]. The BLRT tests whether a model with "K classes" is better than a model with "K-1 classes." A significant BLRT indicates that a model with $\mathrm{K}$ classes is better $[34,35]$. Second, we used non-statistical criteria to select the best model. The addition of one extra class should be conceptually meaningful and represent a subtype that is different from other subtypes in the model with fewer classes [35]. The LCA were performed in Mplus 7.1 [36]. Based on the selected best model, each participant was given a posterior probability of being assigned in each latent class. Each individual was classified into one class according to their highest posterior probability. The class membership for each individual was exported to SPSS to represent subtypes of depression.

To examine whether socio-demographic, medical, and psychological care characteristics were related to distinct subtypes of depression, chi-squared tests and analyses of variance (ANOVAs) were performed on each variable. To examine whether baseline subtypes of depression predicted the longitudinal course of depressive symptoms (based on the 10-item CES-D), the interaction effect between subtypes and time was examined with general linear modeling (GLM, $p<0.05$ ). For patients within each subtype, separate GLMs examined the longitudinal course of depressive symptoms. Cohen's $d$ was calculated to represent the magnitude of change (small effect $d=0.3$, medium effect $d=0.5$, large effect $d>0.8$ ) [37].

\section{Results}

\section{Description of baseline sample}

Table 1 shows the characteristics of the 243 participants. Mean age was 50.91 years, approximately $20 \%$ were male, almost half were highly educated, and about $40 \%$ were diagnosed with breast cancer. Figure 1 presents the mean scores on the 10
CES-D items at baseline. Patients reported relatively high scores (indicating more severe depression) on the items "concentration problems," "everything an effort," and "could not get going" and very low scores on the items "poor appetite" and "life a failure."

\section{Identifying depression subtypes in the 243 participants}

Table 2 presents the fit indices of the examined LCA models. The BIC and entropy favored the three-class model, whereas the AIC favored the four-class model. In addition, both the three- and four-class models had significant BLRT. Thus, from a statistical perspective, both models were acceptable. We then compared the two models from a non-statistical perspective. The three-class model represented three conceptually distinct subtypes of depression, and all contained a substantial number of participants $(47,41$, and $12 \%)$. For the fourclass model, one class of the three-class model (class 2) was separated into two smaller classes, but the two smaller classes showed similar patterns. Thus, the three-class model had a better interpretability and was selected (Fig. 1).

Patients in class $1(47 \%)$ reported an overall low level of depressive symptoms, with elevated scores on concentration problems, sleep problems, and fatigue-related symptoms (everything an effort, could not get going). Patients in class 2 (41\%) reported higher levels of depressive symptoms than class 1 , with similar levels of concentration problems, sleep problems, and fatigue as class 1 and additionally depressed mood ("felt depressed," "felt sad"). Patients in class 3 (12\%) reported an overall high level of depressive symptoms, with elevated scores on depressed mood ("felt blue," felt sad, felt depressed) as well as fatigue and concentration problems.

\section{Predictors of depression subtypes}

None of the socio-demographic, medical, and psychological care characteristics significantly distinguished between the three subtypes of depression (Table 1).

\section{The course of depressive symptoms between distinct subtypes}

A significant interaction between subtypes and time showed that the specific subtype of depression at baseline predicted the longitudinal course of depressive symptoms $\left(F_{\text {subtype }} \times\right.$ time $(4,254)=8.46, p<0.001)$. Patients in class 3 reported the largest improvements in depressive symptoms from T1 to T3; patients in class 2 also reported large improvements, whereas patients in class 1 reported moderate improvements from T1 to T3 (Table 3). Specifically, patients in classes 2 and 3 reported large improvements in depressive symptoms from $\mathrm{T} 1$ to $\mathrm{T} 2$, with no significant changes between $\mathrm{T} 2$ and $\mathrm{T} 3$. In contrast, patients in class 1 reported mainly moderate 
Table 1 Socio-demographic, medical, and psychosocial care characteristics in the entire sample and three subtypes of depressive symptoms $(n=243)$

\begin{tabular}{|c|c|c|c|c|c|}
\hline Predictor & Total sample & Class $1(n=114)$ & Class $2(n=99)$ & Class $3(n=30)$ & \\
\hline & Mean (SD) & Mean (SD) & Mean (SD) & Mean (SD) & ANOVA \\
\hline Age (in years) & $50.91(10.31)$ & $50.64(10.84)$ & $51.28(9.85)$ & $50.66(10.05)$ & $F(2,240)=0.11^{\text {n.s. }}$ \\
\hline Time since diagnosis (in years) & $2.84(4.69)$ & $2.91(5.25)$ & $2.59(4.17)$ & $3.41(4.02)$ & $F(2,233)=0.36^{\text {n.s. }}$ \\
\hline \multirow[t]{2}{*}{ Physical symptoms at $\mathrm{T} 1$} & $7.04(4.60)$ & $6.71(4.28)$ & $6.94(4.71)$ & $8.75(5.21)$ & $F(2,239)=2.27^{\text {n.s. }}$ \\
\hline & $\%$ & $\%$ & $\%$ & $\%$ & $x^{2}$ \\
\hline \multicolumn{6}{|l|}{ Gender } \\
\hline Man & $22.6 \%$ & $21.7 \%$ & $24.2 \%$ & $20.7 \%$ & \multirow[t]{2}{*}{$0.26^{\text {n.s. }}$} \\
\hline Woman & $77.4 \%$ & $78.3 \%$ & $75.8 \%$ & $79.3 \%$ & \\
\hline \multicolumn{6}{|l|}{ Educational level } \\
\hline Low & $25.1 \%$ & $22.1 \%$ & $24.2 \%$ & $40.7 \%$ & \multirow[t]{3}{*}{$5.08^{\text {n.s. }}$} \\
\hline Middle & $28.1 \%$ & $26.5 \%$ & $31.6 \%$ & $22.2 \%$ & \\
\hline High & $46.8 \%$ & $51.3 \%$ & $44.2 \%$ & $37.0 \%$ & \\
\hline \multicolumn{6}{|l|}{ Relationship } \\
\hline Yes & $76.7 \%$ & $72.6 \%$ & $80.2 \%$ & $81.5 \%$ & \multirow[t]{2}{*}{$2.09^{\text {n.s. }}$} \\
\hline No & $23.3 \%$ & $27.4 \%$ & $19.8 \%$ & $18.5 \%$ & \\
\hline \multicolumn{6}{|l|}{ Cancer type } \\
\hline Breast cancer & $41.9 \%$ & $44.7 \%$ & $38.8 \%$ & $41.4 \%$ & \multirow[t]{8}{*}{$16.29^{\text {n.s. }}$} \\
\hline Digestive system cancer & $6.6 \%$ & $7.9 \%$ & $7.1 \%$ & $0.0 \%$ & \\
\hline Lung cancer & $5.4 \%$ & $6.1 \%$ & $5.1 \%$ & $3.4 \%$ & \\
\hline Hematologic cancer & $10.4 \%$ & $6.1 \%$ & $13.3 \%$ & $17.2 \%$ & \\
\hline Head and neck cancer & $7.5 \%$ & $5.3 \%$ & $12.2 \%$ & $0.0 \%$ & \\
\hline Gynecological cancer & $7.9 \%$ & $9.6 \%$ & $6.1 \%$ & $6.9 \%$ & \\
\hline Multiple malignant tumors & $9.5 \%$ & $8.8 \%$ & $9.2 \%$ & $13.8 \%$ & \\
\hline Others & $10.8 \%$ & $11.4 \%$ & $8.2 \%$ & $17.2 \%$ & \\
\hline \multicolumn{6}{|l|}{ Metastases } \\
\hline Yes & $32.1 \%$ & $31.6 \%$ & $29.6 \%$ & $42.9 \%$ & \multirow[t]{2}{*}{$1.78^{\text {n.s. }}$} \\
\hline No & $67.9 \%$ & $68.4 \%$ & $70.4 \%$ & $57.1 \%$ & \\
\hline \multicolumn{6}{|l|}{ Comorbid diseases } \\
\hline Yes & $24.9 \%$ & $26.3 \%$ & $24.0 \%$ & $22.2 \%$ & \multirow[t]{2}{*}{$1.53^{\text {n.s. }}$} \\
\hline No & $75.1 \%$ & $73.7 \%$ & $76.0 \%$ & $77.8 \%$ & \\
\hline \multicolumn{6}{|l|}{ Perceived prognosis } \\
\hline Favorable & $47.3 \%$ & $52.2 \%$ & $42.4 \%$ & $44.8 \%$ & \multirow[t]{3}{*}{$8.87^{\text {n.s. }}$} \\
\hline Unfavorable & $26.3 \%$ & $13.0 \%$ & $11.1 \%$ & $27.6 \%$ & \\
\hline Uncertain & $14.0 \%$ & $22.6 \%$ & $32.3 \%$ & $20.7 \%$ & \\
\hline \multicolumn{6}{|l|}{ Recurrence } \\
\hline Yes & $14.8 \%$ & $12.2 \%$ & $16.2 \%$ & $20.7 \%$ & \multirow[t]{2}{*}{$1.57^{\text {n.s. }}$} \\
\hline No & $85.2 \%$ & $87.8 \%$ & $83.8 \%$ & $79.3 \%$ & \\
\hline \multicolumn{6}{|l|}{ Under medical treatment or not at $\mathrm{T} 1$} \\
\hline Yes & $48.1 \%$ & $46.5 \%$ & $46.6 \%$ & $59.3 \%$ & \multirow[t]{2}{*}{$1.53^{\text {n.s. }}$} \\
\hline No & $51.9 \%$ & $53.5 \%$ & $53.4 \%$ & $40.7 \%$ & \\
\hline \multicolumn{6}{|l|}{ Received type of medical treatment } \\
\hline Operation & $17.3 \%$ & $15.7 \%$ & $16.2 \%$ & $27.6 \%$ & $15.98^{\text {n.s. }}$ \\
\hline Chemotherapy & $9.1 \%$ & $7.0 \%$ & $10.1 \%$ & $13.8 \%$ & \\
\hline Radiotherapy & $2.9 \%$ & $1.7 \%$ & $4.0 \%$ & $3.4 \%$ & \\
\hline Operation + chemotherapy & $19.8 \%$ & $20.9 \%$ & $19.2 \%$ & $17.2 \%$ & \\
\hline Operation + radiotherapy & $16.0 \%$ & $14.8 \%$ & $21.2 \%$ & $3.4 \%$ & \\
\hline Chemotherapy + radiotherapy & $5.8 \%$ & $3.5 \%$ & $8.1 \%$ & $6.9 \%$ & \\
\hline Operation + chemotherapy + radiotherapy & $23.0 \%$ & $28.7 \%$ & $16.2 \%$ & $24.1 \%$ & \\
\hline Others & $6.2 \%$ & $7.8 \%$ & $5.1 \%$ & $3.4 \%$ & \\
\hline Type of psychological care (T1-T2) ${ }^{\mathrm{a}}$ & & & & & \\
\hline Individual & $58.0 \%$ & $61.0 \%$ & $59.4 \%$ & $37.5 \%$ & $12.81^{\text {n.s. }}$ \\
\hline
\end{tabular}


Table 1 (continued)

\begin{tabular}{|c|c|c|c|c|c|}
\hline Predictor & Total sample & Class $1(n=114)$ & Class $2(n=99)$ & Class $3(n=30)$ & \\
\hline Group & $7.0 \%$ & $5.2 \%$ & $9.4 \%$ & $6.3 \%$ & \\
\hline Individual + group & $16.6 \%$ & $14.3 \%$ & $20.3 \%$ & $12.5 \%$ & \\
\hline Other & $1.9 \%$ & $2.6 \%$ & $1.6 \%$ & $0.0 \%$ & \\
\hline Missing & $16.6 \%$ & $16.9 \%$ & $9.4 \%$ & $43.8 \%$ & \\
\hline \multicolumn{6}{|c|}{ Psychological care finished at $\mathrm{T}^{\mathrm{a}}$} \\
\hline Yes & $19.7 \%$ & $15.6 \%$ & $21.9 \%$ & $31.3 \%$ & $2.36^{\text {n.s. }}$ \\
\hline \multicolumn{6}{|c|}{ Type of psychological care (T2-T3) ${ }^{\mathrm{a}}$} \\
\hline $\begin{array}{l}\text { Individual } \\
\text { Group }\end{array}$ & $\begin{array}{l}42.0 \% \\
4.5 \%\end{array}$ & $\begin{array}{l}45.5 \% \\
6.5 \%\end{array}$ & $\begin{array}{l}37.5 \% \\
1.6 \%\end{array}$ & $\begin{array}{l}43.8 \% \\
6.3 \%\end{array}$ & $5.29^{\text {n.s. }}$ \\
\hline Individual + group & $19.7 \%$ & $16.9 \%$ & $25.0 \%$ & $12.5 \%$ & \\
\hline Other & $1.9 \%$ & $2.6 \%$ & $1.6 \%$ & $0.0 \%$ & \\
\hline Missing & $31.8 \%$ & $28.6 \%$ & $34.4 \%$ & $37.5 \%$ & \\
\hline \multicolumn{6}{|c|}{ Psychological care finished at $\mathrm{T}^{\mathrm{a}}$} \\
\hline Yes & $47.1 \%$ & $48.1 \%$ & $43.8 \%$ & $56.3 \%$ & $0.85^{\text {n.s. }}$ \\
\hline
\end{tabular}

${ }^{\mathrm{a}}$ These analyses were conducted in 157 patients who completed the two follow-up measurements

improvements from $\mathrm{T} 1$ to $\mathrm{T} 2$ and small improvements until T3. Additionally, the T1-T2 changes for class 3 were much larger than the T1-T2 changes for class 2.

\section{Discussion}

With the use of a bottom-up, empirically driven approach (LCA), this study identified subgroups of cancer patients with distinct subtypes of depression in cancer patients receiving psychological care at psycho-oncology institutions. Before psychological care, three distinct subtypes of depression were identified: class 1 (47\%), with mild depression, reported mainly concentration problems, sleep problems, and fatigue; class $2(41 \%)$ had slightly higher levels of depression than class 1 , with similar concentration and sleep problems and fatigue as class 1 with additional depressed mood; and class $3(12 \%)$ reporting more severe depression mainly characterized by depressed mood and fatigue and concentration problems. Patients' socio-demographic, medical, and psychological care characteristics (e.g., age, cancer type) did not significantly distinguish these subtypes. However, these subtypes at baseline predicted the courses of depression during psychological care.

The three subtypes differed in the nature of symptoms. Somatic problems (sleep and appetite problems) were not found to be key symptoms differentiating subtypes, although sleep problems were more pronounced in the less-severe subtypes (class 1 and class 2) than in the severe subtype (class 3 ). This is partly in line with a review on empirically driven subtypes of depression, suggesting that somatic symptoms (e.g., fatigue and sleep disturbances) are more common in less-severely depressed patients [11]. In MI patients with MDD, sleep and appetite problems distinguished the moderately from the severely depressed individuals [27]. In our study, as patients did not necessarily meet the diagnostic
Fig. 1 Mean item scores of 10-item CES-D in the three latent classes and the total sample at baseline

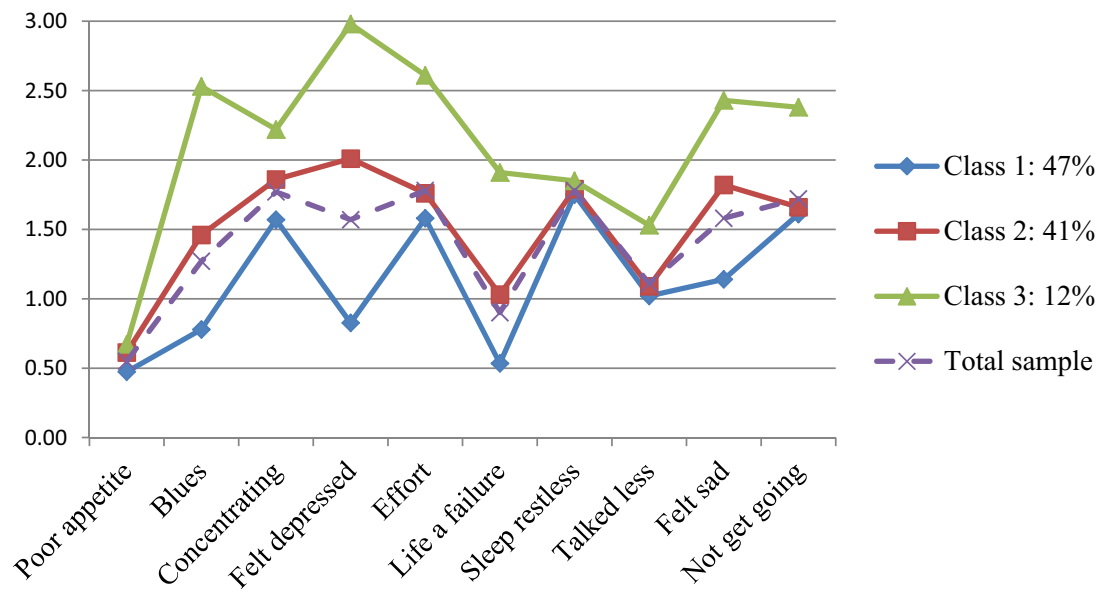


Table 2 Fit indices and class prevalence for the latent class analysis $(n=243)$

\begin{tabular}{|c|c|c|c|c|c|c|c|c|c|}
\hline & \multirow[t]{2}{*}{$\mathrm{BIC}$} & \multirow[t]{2}{*}{ AIC } & \multirow[t]{2}{*}{ Entropy } & \multirow[t]{2}{*}{ BLRT } & \multicolumn{5}{|c|}{ Class size $n(\%)$} \\
\hline & & & & & 1 & 2 & 3 & 4 & 5 \\
\hline 2-class & 5983.43 & 5875.15 & 0.78 & $282.21 * * *$ & $167(69 \%)$ & $76(31 \%)$ & & & \\
\hline 3-class & 5907.58 & 5760.87 & 0.98 & $136.28 * * *$ & $114(47 \%)$ & $99(41 \%)$ & $30(12 \%)$ & & \\
\hline 4-class & 5932.11 & 5746.98 & 0.94 & $35.89^{*}$ & $112(46 \%)$ & $82(34 \%)$ & $29(12 \%)$ & $20(8 \%)$ & \\
\hline 5-class & 5970.30 & 5746.75 & 0.92 & $22.23^{n s}$ & $112(46 \%)$ & $70(29 \%)$ & $29(12 \%)$ & $20(8 \%)$ & $12(5 \%)$ \\
\hline
\end{tabular}

The numbers in bold indicate the best values of each fit indices

$B I C$ Bayesian information criterion, AIC Akaike information criterion, BLRT bootstrapped likelihood ratio test

${ }^{*} p<.05 ; * * * p<.001$

criterion of MDD, we did not include a large group with severely depressed symptoms. This might explain why we found little distinction between the subtypes regarding somatic problems.

Another explanation could be the measure of somatic problems. One study in people with MDD, using a diagnostic interview including a more specific assessment of somatic problems, found three subtypes of depression differentiated by somatic problems [22]. Our study included only two CES-D items on poor appetite and sleep problems, which might have prevented us from highlighting somatic symptoms for differentiating subtypes of depression. Future studies in cancer patients, with a more detailed measurement of somatic problems, are needed to closely examine the role of somatic problems in differentiating depression subtypes.

Our three subtypes of depression varied mainly regarding depressed mood. This is different from findings of a previous study in people with MDD [22]. They found three subtypes of depression (i.e., moderate subtype, severe typical subtype, and severe atypical subtype), of which depressed mood did not distinguish between the three subtypes. In their study, all subtypes reported relatively high scores on depressed mood, which makes sense in people with MDD, as this is one of the core symptoms of depression. Cancer patients in our sample reported elevated levels of depressive symptoms but did not fulfill the criteria for a diagnosis of MDD.

The three subtypes of depression also differed in their severity of depressive symptoms (i.e., low, moderate, and high). This result is in line with a systematic review, reporting that distinct subtypes of depression (as derived by LCA) differed in the severity of depression [11]. Empirical studies using LCA to identify subtypes of depression reported similar findings [16, 19, 22, 23]. For instance, in a study of Hybels et al. in older adults, their three subtypes of depression differed primarily by severity [16]. Unlike our study, the Hybels study did not pre-select participants with elevated depressive symptoms, which might explain why their largest group (59\%) reported almost no depressive symptoms [16]. It seems to suggest that the role of severity does not matter with the sample characteristics (patients with clinically elevated depression or not). Altogether, their findings, together with ours, suggest that LCA not only classifies people based on symptoms but also on severity.

Our three empirically driven subtypes were partly in line with a study on clinically driven subtypes of depression in prostate cancer patients [32]. Their study confirmed the existence of five pre-defined subtypes of depression ("depressed mood," "melancholic," "anhedonic," "somatic," and

Table 3 Changes in the overall levels of 10-item CES-D at each class and the follow-up sample

Changes in overall levels of 10-item CES-D

\begin{tabular}{|c|c|c|c|c|c|c|c|c|c|}
\hline & \multirow{2}{*}{$\begin{array}{l}\mathrm{T} 1 \\
M(\mathrm{SD})\end{array}$} & \multirow{2}{*}{$\begin{array}{l}\mathrm{T} 2 \\
M(\mathrm{SD})\end{array}$} & \multirow{2}{*}{$\begin{array}{l}\mathrm{T} 3 \\
M(\mathrm{SD})\end{array}$} & \multicolumn{2}{|l|}{$\Delta \mathrm{T} 1-\mathrm{T} 2^{\mathrm{a}}$} & \multicolumn{2}{|c|}{$\Delta \mathrm{T} 2-\mathrm{T}^{\mathrm{a}}$} & \multicolumn{2}{|l|}{$\Delta \mathrm{T} 1-\mathrm{T}^{\mathrm{a}}$} \\
\hline & & & & $F$ & $d$ & $F$ & $d$ & $F$ & $d$ \\
\hline Class $1: n=66$ & $11.24(2.92)$ & $9.59(4.11)$ & $8.03(5.16)$ & $9.98 * *$ & 0.46 & $8.44 * *$ & 0.33 & $15.89 * * *$ & 0.77 \\
\hline Class 2: $n=55$ & $14.98(2.84)$ & $10.91(5.81)$ & $9.76(5.41)$ & $29.19 * * *$ & 0.89 & 2.71 & 0.20 & $29.24 * * *$ & 1.20 \\
\hline Class $3: n=12$ & $21.11(2.26)$ & $10.89(5.88)$ & $9.11(6.88)$ & $27.11 * * *$ & 2.29 & 1.43 & 0.28 & $19.76^{* * *}$ & 2.34 \\
\hline Total sample: $n=133$ & $13.51(3.95)$ & $10.24(5.02)$ & $8.84(5.42)$ & $71.24 * * *$ & 0.72 & $6.23 *$ & 0.27 & $62.83 * * *$ & 0.98 \\
\hline
\end{tabular}

${ }^{a}$ As GLM applied a listwise deletion to handle missing values, the GLM were conducted on 133 people with valid data on all CES-D items at three measurements

$* * p<.01 ; * * * p<.001$ 
"cognitive"). Similarly, we found that depressed mood and cognitive problems were important in distinguishing subtypes of depression in cancer patients. However, we did not find evidence supporting the other three subtypes (melancholic, anhedonic, and somatic), which might be due to the lack of assessment of these depression symptoms (e.g., suicidal thoughts, diminished pleasure) in our study.

The three subtypes of depression could not be distinguished by socio-demographic or medical characteristics, which differ from previous findings. In a general population, Hybels et al. identified three subtypes of depression ("severe," "moderate," and "no symptoms"). They found that people with no symptoms were more likely to be younger, male, highly educated, married, and in better physical health than those with severe. They also found that people with moderate were more similar to severe than to people with no symptoms [16]. This finding may explain why we did not find significant correlations with socio-demographic characteristics, as we did not include patients without depressive symptoms. Additionally, although statistically non-significant, patients with the most severe depression (i.e., class 3 ) were more likely to have an unfavorable prognosis, metastases, and/or cancer recurrence. These results seemed to suggest that the more advanced disease might play a role in distinguishing the types of depression. The relatively small number of patients in class 3 might have decreased the power to find significant predictors. Future studies with a larger sample are needed to further examine this issue.

The three subtypes of depression assessed before psychological care predicted the course of depressive symptoms over 9 months. Although all three classes reported improvements in depressive symptoms, they differed in the magnitude of change: class 1 reported moderate improvements, class 2 large improvements, and class 3 the largest improvements. The fact that the most severely depressed patients (i.e., the "severe typical" class) reported more improvements than the other groups is in line with a previous longitudinal study [22]. This study shows that the severe typical group, reporting mainly depressed mood, was the most likely to improve their symptoms. As the three subtypes also differed in their severity of depression, an explanation may be that it is the severity rather than the nature that explains why people with the most severe depression had more room for improvement. The large improvements of this group might reflect a recovery of depression (naturally or due to the received care) or simply statistical regression to mean. As this is the first study examining the longitudinal courses of depressive symptoms reported by cancer patients with distinct subtypes of depression, future studies are needed to replicate our findings and explore the possible reasons for this. Additionally, due to the lack of a control group, it remains unclear about the reasons of these improvements (i.e., participants' attendance of psychological care or other reasons). Future intervention studies with a control group are needed to further explore this issue.

This study suggests that the three subtypes of depression differed by both the nature and severity of depressive symptoms. In practice, these subtypes can be identified before the start of treatment, by systematically examining all depressionrelated symptoms and the most elevated subtype of depression for each individual. This information can be taken into account when psychologists decide on which types of treatment to offer. For example, for patients of class 1, interventions could be focused on cognitive (e.g., concentration problems) and somatic problems (e.g., sleep problems, fatigue), whereas for patients of class 3 , interventions could mainly target depressed mood. These findings suggest that not all cancer patients reporting elevated levels of depressive symptoms (with many different combinations of symptoms possible) should be offered the same type of treatment.

Several limitations should be considered when interpreting the findings of this study. First, two DSM-5 criteria for MDD were not assessed, which may have led to the nonidentification of other subtypes (e.g., melancholic depression). Some studies found that anhedonia (diminished pleasure/interest) could distinguish different subtypes of depression [27], whereas others showed that depressed mood and anhedonia cluster together [22]. Thus, future research should include all core depressive symptoms. Second, this study used a naturalistic design without a control group. The lack of a control group prohibits drawing conclusions about the extent of changes in all subtypes that were due to psychological care or other reasons (e.g., natural adaptation). Third, the sample size of this study was relatively small, which reduced the power to find relevant predictors of subtypes. Fourth, our sample consisted of cancer patients participating in psychological care and included mainly middle-aged women with breast cancer with a high education, similar to other studies [38]. Thus, the generalizability of our findings remains unclear. Specifically, given that our sample were patients seeking help, it is likely that our sample would be at higher risk for clinically elevated depression. This might be the reason for the identification of the classes with moderate to high levels of depression and the moderate- to large-sized improvements in depressive symptoms over time. Future studies on a general cancer population are needed to compare findings.

Notwithstanding these limitations, this study is the first to use a bottom-up, empirically driven approach to identify subtypes of depression in cancer patients. The advanced analytic method provided a more detailed description regarding the heterogeneity of depression in cancer patients. Our findings confirm distinct subtypes of depression in cancer patients with differential courses of depressive symptoms with psychological care over 9 months. The identification of distinct subtypes of depression could serve as a first step to optimize psychological care for depressed cancer patients. Future research is 
needed to examine the care needs of distinct subgroups of depressed cancer patients and their differential responses to distinct interventions for reducing depression.

Acknowledgments The current study was financially supported by the Dutch Pink Ribbon Foundation and the Ingeborg Douwes Stichting. We would also like to give our appreciation to all the IPSO institutions for participating in the current study.

Open Access This article is distributed under the terms of the Creative Commons Attribution-NonCommercial 4.0 International License (http://creativecommons.org/licenses/by-nc/4.0/), which permits any noncommercial use, distribution, and reproduction in any medium, provided you give appropriate credit to the original author(s) and the source, provide a link to the Creative Commons license, and indicate if changes were made.

\section{References}

1. American Psychiatric Association (2013) Diagnostic and statistical manual of mental disorders. American Psychiatric Publishing, Arlington

2. Krebber AMH, Buffart LM, Kleijn G, Riepma IC, de Bree R, Leemans CR, Becker A, Brug J, van Straten A, Cuijpers P, Verdonck-de Leeuw IM (2014) Prevalence of depression in cancer patients: a meta-analysis of diagnostic interviews and self-report instruments. Psycho-Oncology 23:121-130

3. Mitchell AJ, Chan M, Bhatti H, Halton M, Grassi L, Johansen C, Meader N (2011) Prevalence of depression, anxiety, and adjustment disorder in oncological, haematological, and palliative-care settings: a meta-analysis of 94 interview-based studies. Lancet Oncol $12: 160-174$

4. Lloyd-Williams M, Dennis M, Taylor F (2004) A prospective study to determine the association between physical symptoms and depression in patients with advanced cancer. Palliat Med 18:558-563

5. DiMatteo M, Lepper H, Croghan T (2000) Depression is a risk factor for noncompliance with medical treatment - meta-analysis of the effects of anxiety and depression on patient adherence. Arch Intern Med 160:2101-2107

6. Jayadevappa R, Malkowicz SB, Chhatre S, Johnson JC, Gallo JJ (2012) The burden of depression in prostate cancer. PsychoOncology 21:1338-1345

7. Harald B, Gordon P (2012) Meta-review of depressive subtyping models. J Affect Disord 139:126-140

8. Baumeister H, Parker G (2010) A second thought on subtyping major depression. Psychother Psychosom 79:388-389

9. Parker G (2006) Through a glass darkly: the disutility of the DSM nosology of depressive disorders. Can J Psychiatry 51:879-886

10. Parker G (2005) Beyond major depression. Psychol Med 35:467474

11. van Loo HM, de Jonge P, Romeijn J, Kessler RC, Schoevers RA (2012) Data-driven subtypes of major depressive disorder: a systematic review. BMC Med 10:156

12. Parker G, Roy K, Wilhelm K, Mitchell P, Austin M, Hadzi-Pavlovic D, Little C (1999) Sub-grouping non-melancholic depression from manifest clinical features. J Affect Disord 53:1-13

13. Rush AJ (2007) The varied clinical presentations of major depressive disorder. J Clin Psychiatry 68:4-10

14. Uher R, Muthen B, Souery D, Mors O, Jaracz J, Placentino A, Petrovic A, Zobel A, Henigsberg N, Rietschel M, Aitchison KJ, Farmer A, McGuffin P (2010) Trajectories of change in depression severity during treatment with antidepressants. Psychol Med 40: 1367-1377

15. Rodgers $\mathrm{S}$, Holtforth MG, Mueller M, Hengartner MP, Roessler W, Ajdacic-Gross V (2014) Symptom-based subtypes of depression and their psychosocial correlates: a person-centered approach focusing on the influence of sex. J Affect Disord 156:92-103

16. Hybels CF, Landerman LR, Blazer DG (2013) Latent subtypes of depression in a community sample of older adults: can depression clusters predict future depression trajectories? J Psychiatr Res 47: $1288-1297$

17. Lee C, Leoutsakos J, Lyketsos CG, Steffens DC, Breitner JCS, Norton MC (2012) Latent class-derived subgroups of depressive symptoms in a community sample of older adults: the cache county study. Int J Geriatr Psychiatry 27:1061-1069

18. Hybels CF, Blazer DG, Pieper CF, Landerman LR, Steffens DC (2009) Profiles of depressive symptoms in older adults diagnosed with major depression: latent cluster analysis. Am J Geriatr Psychiatr 17:387-396

19. Carragher N, Adamson G, Bunting B, McCann S (2009) Subtypes of depression in a nationally representative sample. J Affect Disord 113:88-99

20. Herman KC, Ostrander R, Walkup JT, Silva SG, March JS (2007) Empirically derived subtypes of adolescent depression: latent profile analysis of co-occurring symptoms in the treatment for adolescents with depression study (TADS). J Consult Clin Psychol 75: 716-728

21. Chen L, Eaton W, Gallo J, Nestadt G (2000) Understanding the heterogeneity of depression through the triad of symptoms, course and risk factors: a longitudinal, population-based study. J Affect Disord 59:1-11

22. Lamers F, Rhebergen D, Merikangas KR, de Jonge P, Beekman ATF, Penninx BWJH (2012) Stability and transitions of depressive subtypes over a 2-year follow-up. Psychol Med 42:2083-2093

23. Lamers F, de Jonge P, Nolen WA, Smit JH, Zitman FG, Beekman ATF, Penninx BWJH (2010) Identifying depressive subtypes in a large cohort study: results from the Netherlands study of depression and anxiety (NESDA). J Clin Psychiatry 71:1582-1589

24. Raison C, Miller A (2003) Depression in cancer: new developments regarding diagnosis and treatment. Biol Psychiatry 54:283-294

25. Newport D, Nemeroff C (1998) Assessment and treatment of depression in the cancer patient. J Psychosom Res 45:215-237

26. Azevedo RM, Roest AM, Hoen PW, de Jonge P (2014) Cognitive/ affective and somatic/affective symptoms of depression in patients with heart disease and their association with cardiovascular prognosis: a meta-analysis. Psychol Med 44:2689-2703

27. Groenewold NA, Doornbos B, Zuidersma M, Vogelzangs N, Penninx BWJH, Aleman A, de Jonge P (2013) Comparing cognitive and somatic symptoms of depression in myocardial infarction patients and depressed patients in primary and mental health care. PLoS One 8, e53859

28. Dehaes JCJM, Vanknippenberg FCE, Neijt JP (1990) Measuring psychological and physical distress in cancer patients - structure and application of the Rotterdam symptom checklist. Br J Cancer 62:1034-1038

29. Radloff LS (1977) The CES-D scale: a self-report depression scale for research in the general population. Appl Psychol Meas 1:385401

30. Schroevers M, Sanderman R, van Sonderen E, Ranchor A (2000) The evaluation of the center for epidemiologic studies depression (CES-D) scale: depressed and positive affect in cancer patients and healthy reference subjects. Qual Life Res 9:1015-1029

31. Schroevers M, Ranchor A, Sanderman R (2003) Depressive symptoms in cancer patients compared with people from the general population: the role of sociodemographic and medical factors. J Psychosoc Oncol 21:1-26 
32. Sharpley CF, Bitsika V, Christie DRH (2013) The incidence and causes of different subtypes of depression in prostate cancer patients: implications for cancer care. Eur J Cancer Care 22:815-823

33. Asparouhov T, Muthén B (2013) Auxiliary variables in mixture modeling: A 3-step approach using Mplus No. 15

34. Jung T, Wickrama KAS (2008) An introduction to latent class growth analysis and growth mixture modeling 2:302-317

35. Nylund KL, Asparoutiov T, Muthen BO (2007) Deciding on the number of classes in latent class analysis and growth mixture modeling: a Monte Carlo simulation study. Struct Equ Modeling 14:535-569

36. Muthén, L.K. and Muthén, B.O. (1998-2012) Mplus user's guide. Seventh Edition

37. Cohen J (1988) Statistical power analysis for the behavioral sciences. Second edition. Lawrence Erlbaum Associates

38. Nekolaichuk CL, Cumming C, Turner J, Yushchyshyn A, Sela R (2011) Referral patterns and psychosocial distress in cancer patients accessing a psycho-oncology counseling service. Psycho-Oncology $20: 326-332$ 